\title{
WAREHOUSE REDESIGNING IN A THREE-ECHELON SUPPLY CHAIN NETWORK WITH CONSIDERATION OF ROUTING UNDER UNCERTAINTY: A LIGHT ROBUST APPROACH
}

\author{
Zahra Azadehranjbar ${ }^{1}$, Ali Bozorgi-Amiri ${ }^{1, *}$ And Arash Zandi ${ }^{2}$
}

\begin{abstract}
This paper addresses the problem of redesigning a three-echelon supply chain network under uncertainty. Since one of the most realistic problems that supply chains are dealt with is routing of vehicles, routing constraints with a split delivery condition are considered in our proposed model. Also, the possibility of outsourcing is considered in order to satisfy demands that exceed the production capacity. Furthermore, in order to deal with the presence of uncertainty in the problem, a light robust approach is developed. The performance of the proposed model is illustrated using a simulation procedure. Sensitivity analysis on the proposed model is also presented in the paper. The results show that the proposed method has a better performance than Light Robust approach and can be used as a useful managerial tool in redesign problems.
\end{abstract}

Mathematics Subject Classification. 90C90.

Received May 6, 2018. Accepted June 20, 2019.

\section{INTRODUCTION}

Supply chain network design (SCND) is a strategic planning process for optimizing the configuration of a supply chain network. In broad terms, SCND involves finding the optimal location and number of facilities (e.g., factories and warehouses), and determining the flow of products across the supply chain so that customer demands are satisfied at minimum cost or maximum profit.

Due to real business requirements, a company may consider either redesigning an existing supply chain in order to adapt it to new business situations or to meet new strategic objectives. Supply chain network redesign (SCNR) is generally triggered by changing market and business situations, often in regard to increased cost pressure and service requirements. For instance, some changes in the demand of different markets, customer and supplier's zones, regional taxes and government rules, etc., turn the optimality of supply chain network. These conditions oblige companies to develop or redesign their supply chain configuration. Due to the rapid changes of conditions in today's world, a company may wish to evaluate to what degree its existing supply chain network deviates from the optimal configuration. Therefore, supply chain network redesign is a strategic planning process for optimizing an existing supply chain and which should consider current locations, constraints and properties

Keywords. Supply chain network redesign, routing, light robust optimization, uncertainty - outsourcing.

1 School of Industrial Engineering, College of Engineering, University of Tehran, Tehran, Iran.

2 Department of Logistics, Wroclaw University of Economics, Wroclaw, Poland.

*Corresponding author: alibozorgi@ut.ac.ir 
with respect to finding a gradual process for a transition from the current condition to the optimal configuration. Thus, redesigning a supply chain is more complex compared to designing a new supply chain network.

During the redesign process, some old facilities may be closed and some new ones may be opened. Due to the manager's decision, some facilities can be consolidated, meaning that the capacity of some facilities may be moved to others. The word capacity has a vast domain of meaning for this situation. It can include all the physical capacities, equipment, human resources or the production power. The possibility of consolidation can help the model to use this capacity in some other places.

Since warehouses' redesigning has much more benefits in an operating supply chain, redesign projects in the warehouses network are more common compared to other layers of supply chain. Ballou and Masters [2] reported that, out of 200 logistic networks, $65 \%$ of them will redesign their warehouse networks. Consolidation and phase-out activities in the warehouse network can save transportation, inventory and warehousing costs [22].

Melachrinoudis and Min [21] introduced a multi-objective model to relocate a single facility. Capacity can be transferred from a source facility to a destination. However, the increase or decrease is not considered for the total network capacity, nor is uncertainty. Finally, a resulting mixed integer linear program (MILP) was solved using commercial software. Melo et al. [25] presented a fairly comprehensive model in this scope. The strategic redesign decisions are not limited to a single layer of the supply chain. The capacity of the facilities can be partially transferred, as an integer or in continuous manner, to another one. The total network capacity can be extended or decreased. Another trait is the consideration of budget limitation on redesign decisions. Again, the uncertainty has not been considered. The authors did not suggest a solution method for their MIP model in this paper, but they proposed some heuristic solution methods in their next articles [23, 24].

Melachrinoudis and Min [22] introduced a warehouse network redesign problem. The strategic redesign decisions contains the phase-out, consolidation of existing warehouses and establishing new sites, and the capacity could be totally transferred. The relocation decisions are considered simultaneously for multi-facilities. There is a limitation on the distance between the warehouse and the customer. Hence, redesign decisions must be made so as not to disrupt the ability to meet the customer's demands. Finally, the authors solved this MILP model by partially relaxing the allocation integer variables combined with rounding procedures.

Martins et al. [20] addressed the problem of redesigning a humanitarian supply chain network for the collection of donated food products and their distribution to non-profit organizations that provide food assistance to the needy population. Their redesign includes opening, closing and expansion in the food bank capacities. They proposed a mathematical model for this multi echelon, multi-product and multi-period problem. . This model had three objectives involved economic, environmental and social objectives and they considered different types of capacitated vehicles for their direct transportations from origins to destinations.

Lee et al. [18], Bing et al. [7], Khatami et al. [15] and Feitó-Cespón et al. [10] used redesigning in reverse and close loop supply chains in different conditions. For example, Feitó-Cespón et al. [10] have used redesign problem on a sustainable reverse supply chain. Development of this study can be seen in some of other works $[8,28,30]$.

On the other hand, different sources of uncertainty exist in reality, such as inaccurate information, vagueness in data and variability [16]. So, many researchers have used uncertain data in their papers and they have applied different methods to solve uncertain SCND problems. Govindan et al. [9] have provided a comprehensive review on SCND papers under uncertainty and existing optimization techniques for dealing with uncertainty.

A review by Klibi et al. [17] demonstrates that "stochastic programming" has been the predominant method to tackle uncertain models in the past researches. Also, Scenario-based stochastic programming have used in many supply chain design and redesign problems. For instance, Bashiri and Rezaei [4] and Kiya and Davoudpour [16] separately considered uncertainty and scenario based stochastic programming in supply chain redesign, and used Sample Average Approximation (SAA) approach together with Benders decomposition as a solution method. Fattahi et al. [10] studied a multi-product supply chain redesign problem in which customer zones' demands were stochastic and price-dependent. Under a multi-period planning horizon, a multi-stage stochastic program was developed for the SCNR problem. 
Despite of wide use of stochastic method by researchers in recent years, this method has shown significant limitations in reality. For example, stochastic programming approaches usually require the availability of probability distributions of random variables such as the probability of an interruption occurrence and its measure of impact. Such historical data, particularly for some rare events, is limited or non-existent. So it is difficult to define the true probability distribution of uncertain parameters [34].

One of other important issues affecting a supply network optimality, is to design a proper vehicle routing plan to supply the demands of each echelon of the chain. Regarding to the literature, although no research has been done in redesigning a supply chain network with consideration of routing, the cutting edge researches in supply chain network design demonstrate the importance of this issue in developing a proper supply network plan. In recent years many researchers considered routing as a vital problem of real supply chains which is so effective in total cost, routing and location decisions. Thus, many papers have considered routing as important as including the uncertainty in the problem and solved the mathematical models based on these considerations. For instance, recently Fazayeli et al. [11] addressed a distribution system including location and routing decisions in a time window to satisfy customers. They considered demand as fuzzy numbers. Also, Majidi et al. [19] considered a fuzzy green routing problem in a pickup and delivery system. Another paper published recently belongs to Rabbani et al. [29] which tried to manage stochastic amount of hazardous wastes while deciding for location, routing and inventory problems. Asadi et al. [1] considered a stochastic multi objective distribution system with economic and environmental objects. Schiffer and Walther [31] used another type of uncertainty and presented a robust location-routing approach to design electric logistics fleets in a real case. More recent researches about uncertain supply chain designs can be found in other papers such as Nadizadeh and Kafash [27], Jouzdani et al. [14], Bashiri and Ghasemi [3], Tönissen and Arts [33].

More recently, robust supply chain design models have been proposed to overcome this drawback, and it can be a good method for many problems in supply chain design and redesigns. The most important reason for this result returns to the type of our decision making. Due to the difficulty and expensive cost of a supply chain redesign, such decisions are to be a long-term decision which cannot change by every small changes in situation. Thus, a robust method which can consider different possible circumstance of coming periods, can be a good candidate. As mentioned above, in stochastic programming ones require the availability of probability distributions of nonpredetermined parameters. However, in many cases it is not possible to obtain such distributions. On the other hand, fuzzy methods are not suitable for the problem investigating in this paper because uncertain parameters are quantitative and the reason of uncertainty existed is unpredictability of them in the decision period.

The problems presented in the redesign literature have two approaches to consider transportation costs:

- Considering a direct distance and definite cost between the warehouse and the customer.

- Considering a coverage matrix with a definite radius to find proper allocation of customers to warehouses.

These approaches are not useful in real cases, because making a tour of deliveries is more cost-effective than single routes. Hence, companies prefer to use a vehicle for a group of customers instead of a single customer. On the other hand, routing can obviously affect the relocation of the warehouses, since defining the routes can decrease the force of proximity to some customers and it helps with the problem of finding the best warehouse network in a fewer number of warehouse zones. Indeed, one of the basic reasons of redesigning supply chain networks is related to this issue that previous strategic decisions may impose a big amount of operational costs such as routing costs due to changing in demands and/or network. Thus, it is important to consider these operational decisions during supply chain redesign and combine them to have complete participation in terms of optimizing future costs.

In this paper, an integrated model of redesign has been developed considering routing, capacity constraints, and outsourcing in redesign decisions under uncertain situations. In this network redesign, based on warehouse's capacity and location and also the location of customers and suppliers, some warehouses can be closed, opened or consolidated with each other to have an optimized network. Considering routings in the network, based on cost and capacity of vehicles, lead to finding optimal allocations of vehicles to customers. Another equally important part of this paper is concerned with the issue that considering the capacity of plants helps managers to use 
other sources such as the market to satisfy customer's demands to avoid losing them. To deal with the uncertain concept, we adapted the light robust formulation with interval data uncertainty and developed a revised light robust model, which will be shown to provide better solutions for uncertain situations. The objective is to minimize the sum of fixed, production, outsourcing, warehousing, transportation, relocation, and consolidation costs.

Based on the literature review provided above, in this paper, the following contributions can be considered:

- Considering routing effects on supply chain network redesign.

- Using a revised light robust approach to handle the magnitude of conservativeness in uncertain model.

In order to enhance the practicality of the model, the following features are considered in mathematical modeling:

- The possibility of outsourcing for the condition that total demand exceeds the production capacity.

- Customer's demand can be split among several vehicles (split delivery routing).

- Capacitated and heterogeneous fleet of transportation vehicle for products delivery.

The rest of this paper is organized as follows: In the next section, we propose a deterministic model for the redesign of a warehouse with respect to routing and capacity constraints. In Section 3, we describe the light robust method. Following that, a revision on the light robust model is developed in order to optimize the uncertain redesign model. Section 4 presents an experimental example generated and solved using random test problems. Also, two types of sensitivity analyses are done. The first one proves that the proposed revision on the light robust model makes a better solution in comparison with the classic light robust model. The other analysis is done to observe the balance of robustness and the quality of solution. Finally, Section 5 draws some conclusions and suggestions for future studies.

\section{Problem Description}

In the current study, we considered a multi-product and three-echelon logistics network comprising manufacturing plants, warehouses and customer zones. The main assumptions are based on Melachrinoudis and Min [22].

The network encounters production capacity, thus we have to outsource a portion of products to satisfy all demands. The purpose of this problem is to consolidate capacity of available warehouses, and select the appropriate sites to open new warehouses or close some old warehouses to reach an optimal network and to determine the vehicle routes and quantity of products transported between facilities to minimize the total costs, including transportation, warehousing, procurement and infrastructure costs involving fixed, relocation and capacity costs.

\section{Assumptions}

1. When a warehouse is consolidated into another warehouse, its whole capacity is relocated.

2. The whole capacity of every existing warehouse can be transferred to every new or existing warehouse that is established or will be remained [22].

3. There are various kinds of products and all of the plants can produce all or some of them within their production capacity.

4. Outsourced demands are transferred to the warehouses directly.

5. There is a heterogeneous fleet for transportation, including some vehicles with different sizes of capacity and different variable and fixed costs.

6. There is a split delivery system so that the demand of each customer can be satisfied from different warehouses.

7. Outsourcing cost for one unit of product type $p$ includes all of its procurement costs involving purchasing, transportation and fixed costs per unit. 


\subsection{Deterministic supply chain network redesign with routing consideration (DSCNRR)}

In this section, we introduce a mathematical model for a comprehensive SCNR problem regarding routing decisions.

\begin{tabular}{|c|c|}
\hline \multicolumn{2}{|c|}{ Sets and indices } \\
\hline$F$ & Set of manufacturing plants indexed by $i, j$ \\
\hline$W_{e}$ & Set of existing warehouses indexed by $i, j$ \\
\hline$W_{n}$ & Set of potential sites for new warehouses indexed by $i, j$ \\
\hline$W$ & Set of all of warehouses; $w=w_{e} \cup w_{n}$; indexed by $i, j$ \\
\hline$K$ & Set of customers indexed by $i, j$ \\
\hline$i, j$ & Indices of all of nodes; $(i, j \in F \cup W \cup K)$ \\
\hline$V$ & Set of vehicles indexed by $v$ \\
\hline$P$ & Set of products indexed by $p$ \\
\hline \multicolumn{2}{|c|}{ Parameters } \\
\hline$d_{i p}$ & Demand of customer $i(i \in K)$ for product $p$ \\
\hline $\operatorname{cap}_{i p}$ & The capacity of warehouse $i(i \in W)$ for product $p$ \\
\hline$M p_{p i}$ & Production capacity of manufacturing plant $i(i \in F)$ for product $p$ \\
\hline $\operatorname{vcap}_{v}$ & The capacity of vehicle $v$ \\
\hline$v c_{v}$ & Variable transportation cost per unit distance for vehicle $v$ \\
\hline$u c_{v}$ & Fixed transportation cost for vehicle $v$ per trip \\
\hline$p c_{p}$ & Production cost for product type $p$ per unit product \\
\hline$o c_{p}$ & Outsourcing cost for product type $p$ per unit product \\
\hline$h c_{i, p}$ & $\begin{array}{l}\text { Inventory holding cost in warehouse } i(i \in W) \text { for product type } p \text { per unit product } \\
\text { per period }\end{array}$ \\
\hline$r_{i j}$ & $\begin{array}{l}\text { Total cost of relocation or consolidation of existing warehouse } i \text { to a new site or } \\
\text { other existing warehouse } j \text {, if } i=j \text { then } r_{i, j}=0\left(i \in W_{e}, j \in W\right)\end{array}$ \\
\hline$f c_{i p}$ & Unit maintenance cost of the capacity of warehouse $i(i \in W)$ for product $p$ \\
\hline$f m_{i}$ & Fix part of the maintenance cost for warehouse $i(i \in W)$ \\
\hline$f n_{i}$ & Cost savings resulting from the capacity transition of existing warehouse $i\left(i \in W_{e}\right)$ \\
\hline$f s_{i}$ & Cost savings resulting from the phase-out of existing warehouse $i\left(i \in W_{e}\right)$ \\
\hline$f b_{i}$ & Fixed cost of building a new warehouse $i\left(i \in W_{n}\right)$ \\
\hline $\operatorname{dis}_{i j}$ & Distance between nodes $i, j(i, j \in F \cup W \cup K)$ \\
\hline \multicolumn{2}{|c|}{ Decision variables } \\
\hline$Q_{i j p v}^{1}$ & $\begin{array}{l}\text { Amount of product p transported by vehiclevfrom nodeito node } j \text { in the first } \\
\text { echelon (plant-warehouse) }(i, j \in F \cup W)\end{array}$ \\
\hline$Q_{i j p v}^{2}$ & $\begin{array}{l}\text { Amount of product } p \text { transported by vehicle } v \text { from node } i \text { to node } j \text { in the second } \\
\text { echelon (warehouse-customer) }(i, j \in W \cup K)\end{array}$ \\
\hline$Y_{i p}$ & Amount of product $p$ outsourced for warehouse $i(i \in W)$ \\
\hline$X_{i j v}^{1}$ & $\begin{array}{l}\text { A binary variable equal to } 1 \text { if arc }(i, j) \text { in the first echelon is visited by vehicle } v \text {; } \\
0 \text {, otherwise. }(i, j \in F \cup W)\end{array}$ \\
\hline$X_{i j v}^{2}$ & $\begin{array}{l}\text { A binary variable equal to } 1 \text { if arc }(i, j) \text { in the second echelon is visited by vehicle } \\
v ; 0 \text {, otherwise. }(i, j \in W \cup K)\end{array}$ \\
\hline$Z_{i j}$ & $\begin{array}{l}\text { A binary variable equal to } 1 \text { if the capacity of warehouse } i\left(i \in W_{e}\right) \text { is transferred } \\
\text { to warehouse } j(j \in W, i \neq j) \text { or remains open }(i=j) \text {, or new warehouse } i(i \in \\
\left.W_{n}, i=j\right) \text { is established; } 0 \text {, otherwise }\end{array}$ \\
\hline
\end{tabular}

The deterministic supply chain network redesign model is as follows:

$\min \sum_{i \in W_{e}} \sum_{j \in W} r_{i j} Z_{i j}+\sum_{i \in W_{n}} f b_{i} Z_{i i}+\sum_{p \in P} \sum_{j \in W} f c_{j p} \sum_{i \in W_{e}} \operatorname{cap}_{i p} Z_{i j}+\sum_{j \in W} f m_{j} Z_{j j}-\sum_{i \in W_{e}}\left[f s_{i}\left(1-\sum_{j \in W} Z_{i j}\right)+f n_{i} \sum_{\substack{j \in W \\ i \neq j}} Z_{i j}\right]$ 
$+\sum_{i \in F} \sum_{j \in W} \sum_{v \in V} u c_{v} X_{i j v}^{1}+\sum_{i, j \in F \cup W} \sum_{v \in V} v c_{v} \operatorname{dis}_{i j} X_{i j v}^{1}+\sum_{i \in W} \sum_{j \in K} \sum_{v \in V} u c_{v} X_{i j v}^{2}+\sum_{i, j \in W \cup K} \sum_{v \in V} v c_{v} \operatorname{dis}_{i j} X_{i j v}^{2}$

$+\sum_{i \in F} \sum_{j \in W} \sum_{p \in P} \sum_{v \in V} p c_{p} Q_{i j p v}^{1}+\sum_{i \in W} \sum_{p \in P} o c_{p} Y_{i p}+\sum_{i \in W} \sum_{j \in K} \sum_{p \in P} \sum_{v \in V} h c_{i p} Q_{i j p v}^{2}$.

Subject to:

$$
\begin{array}{ll}
\sum_{i \in W} Z_{i j} \leq|W| Z_{j j} & \forall j \in W \\
\sum_{j \in W} Z_{i j} \leq 1 & \forall i \in W \\
\sum_{j \in W} Z_{i j} \leq 0 & \forall i \in W_{n} \\
i \neq j & \\
\sum_{j \in K} \sum_{v \in V} Q_{i j p v}^{2} \leq \sum_{i / \in W} \operatorname{cap}_{i / p} Z_{i / i} & \forall i \in W, \forall p \in P
\end{array}
$$

first echelon constraints

$$
\begin{array}{ll}
\sum_{j \in W} \sum_{v \in V} Q_{i j p v}^{1} \leq M P_{i p} & \forall i \in F, \forall p \in P \\
\sum_{p \in P} Q_{i j p v}^{1} \leq \operatorname{vcap}_{v} X_{i j v}^{1} & \forall i \in F \cup W, \forall j \in W, \forall v \in V \\
\sum_{i \in F} \sum_{j \in W} X_{i j v}^{1} \leq 1 & \forall v \in V \\
\sum_{j \in F \cup W} X_{i j v}^{1}=\sum_{j \in F \cup W} X_{j i v}^{1} & \forall i \in F \cup W, \forall v \in V \\
\sum_{v \in V} \sum_{i \prime \in F \cup W} Q_{i \prime i p v}^{1}-\left(\sum_{v \in V} \sum_{j \in K} Q_{i j p v}^{2}-Y_{i p}\right)=\sum_{v \in V} \sum_{i \prime \in F \cup W} Q_{i i / p v}^{1} & \forall i \in W, \forall p \in P \\
\sum_{i \in F \cup W} Q_{i j p v}^{1} \geq \sum_{i \in F \cup W} Q_{j i p v}^{1} & \forall j \in W, \forall p \in P, \forall v \in V
\end{array}
$$

second echelon constraints

$$
\begin{array}{ll}
\sum_{p \in P} Q_{i j p v}^{2} \leq \operatorname{vcap}_{v} X_{i j v}^{2} & \forall i \in W \cup K, \forall j \in K, \forall v \in V \\
\sum_{i \in W} \sum_{j \in K} X_{i j v}^{2} \leq 1 & \forall v \in V \\
\sum_{j \in W \cup K} X_{i j v}^{2}=\sum_{j \in W \cup K} X_{j i v}^{2} & \forall i \in W \cup K, \forall v \in V \\
\sum_{v \in V} \sum_{j \in W \cup K} Q_{j i p v}^{2}-d_{i p}=\sum_{v \in V} \sum_{j \in W \cup K} Q_{i j p v}^{2} & \forall i \in K, \forall p \in P \\
\sum_{i \in W \cup K} Q_{i j p v}^{2} \geq \sum_{i \in W \cup K} Q_{j i p v}^{2} & \forall j \in K, \forall p \in P, \forall v \in V \\
Q_{i j p v}^{1}, Q_{i j p v}^{2}, Y_{i p} \geq 0 & \\
X_{i j v}^{1}, X_{i j v}^{2}, Z_{i j} \quad \text { binary variables. } &
\end{array}
$$


Objective function (2.1) includes the costs and cost savings consisting of transportation, warehousing, outsourcing, producing, establishing and phase-out and consolidation of warehouses. If an existing warehouse is phased out or consolidated to another warehouse, a cost saving occurs. In this paper, unlike previous studies, for transferred warehouses, a profit parameter apart from the cost saving is defined $\left(f n_{i}\right)$. This profit parameter includes the profits made by selling the warehouse and similar assets.

Constraint (2.2) ensures that the capacity of existing warehouses cannot be consolidated into a closed or unestablished warehouse. Constraint (2.3) considers the various alternatives for existing warehouse $j$. Constraint (2.4) ensures that the capacity of new warehouses cannot be transferred. Constraint (2.5) ensures the total product $p$ that flows from warehouse $i$ to the set of customers covered by this warehouse cannot be larger than the installed capacity.

Constraints (2.6)-(2.11) are related to the first echelon of supply chain (plant-warehouse). Constraint (2.6) limits the total product flow from facility $i \in F$ to its capacity. Constraint (2.7) guarantees that the vehicle's capacity should not be exceeded. It, also, implies that the quantity of product type ptransported by vehicle type $v$ through arc $(i, j)$ can be positive if only the $\operatorname{arc}(i, j)$ is visited by that vehicle $\left(X_{i j v}=1\right)$. Constraint (2.8) ensures a vehicle cannot be employed by multiple plants. Constraint (2.9) implies that every point entered by the vehicle should be left by the vehicle. Constraint (2.10) is the balance constraint for product type $p$ in warehouse $i$. Amount of product $p$ left warehouse $i$ is equal to amount of product $p$ entered to this warehouse minus amount of product $p$ that should be remained there. Constraint (2.11) is a logical constraint that is created for preventing the vehicles to pick up products from warehouse zones.

Constraints (2.12)-(2.16) are similar to constraints (2.7)-(2.11) in the second echelon of supply chain (warehouse-customer). Finally, constraints (2.17) and (2.18) display nonnegative and binary variables.

\section{RoBUst APPROACH}

Robust optimization is one of the techniques to tackle uncertain issues. It is a strong methodology to find a robust solution and manage the risks arising from noisy data [26].

The robust formulation with interval data uncertainty was first introduced in mathematical programming by Soyster [32]. Soyster considered the uncertain technological coefficients in the standard form of linear mathematical programming. In this methodology, the maximum level of conservatism is incorporated in the model by substituting the supremum values, or worst-case values, of the uncertain technological coefficients. The drawback of Soyster's approach is that the robust solution can be highly over- conservative in practical cases because the probability at which uncertain parameters reach their worst values is as low as they reach their normal values [34]

A linear robust optimization approach in continuous spaces for interval uncertain parameters is also proposed by Bertsimas and Sim [5], Bertsimas and Sim [6]. They argued that in real conditions it is less probable that all uncertain parameters take their worst-case value. These robust optimization approaches sometimes lead to very conservative solutions, so Fischetti and Monaci [13] investigated a new way to model the uncertainty, leading to a modelling framework, called Light Robustness (LR). This approach is a balancing between the feasibility due to uncertain parameters (solution robustness) and the quality of the solution. They proposed two models as light robust approach and their first model is a strongly relied on the Bertsimas and Sim definition of robustness. We will explain a summary of this approach in follows.

\subsection{The Light Robustness approach (LR approach)}

In Bertsimas and Sim (BS) approach, due to the objective function value, the optimal solution can be worse than the optimal solution of the deterministic problem. It should be mentioned that LR approach is concentrated on balancing the quality of the solution (the objective function value) and the robustness of the solution (the uncertainty of the coefficients). This method, as a new process of formulation, finds the most robust solution which is not too far from the nominal (deterministic) optimal solution. 
Let us consider a linear mathematical programming model as:

$$
\begin{array}{ll}
\min \sum_{i, j} c_{j} X_{j} & \\
\text { s.t.: } & \\
\sum_{j \in N} a_{i j} X_{j} \leq b_{i} & \forall i \in M \\
X_{j} \geq 0 & \forall j \in N
\end{array}
$$

The counterpart of (3.1)-(3.3) in LR can be shown as:

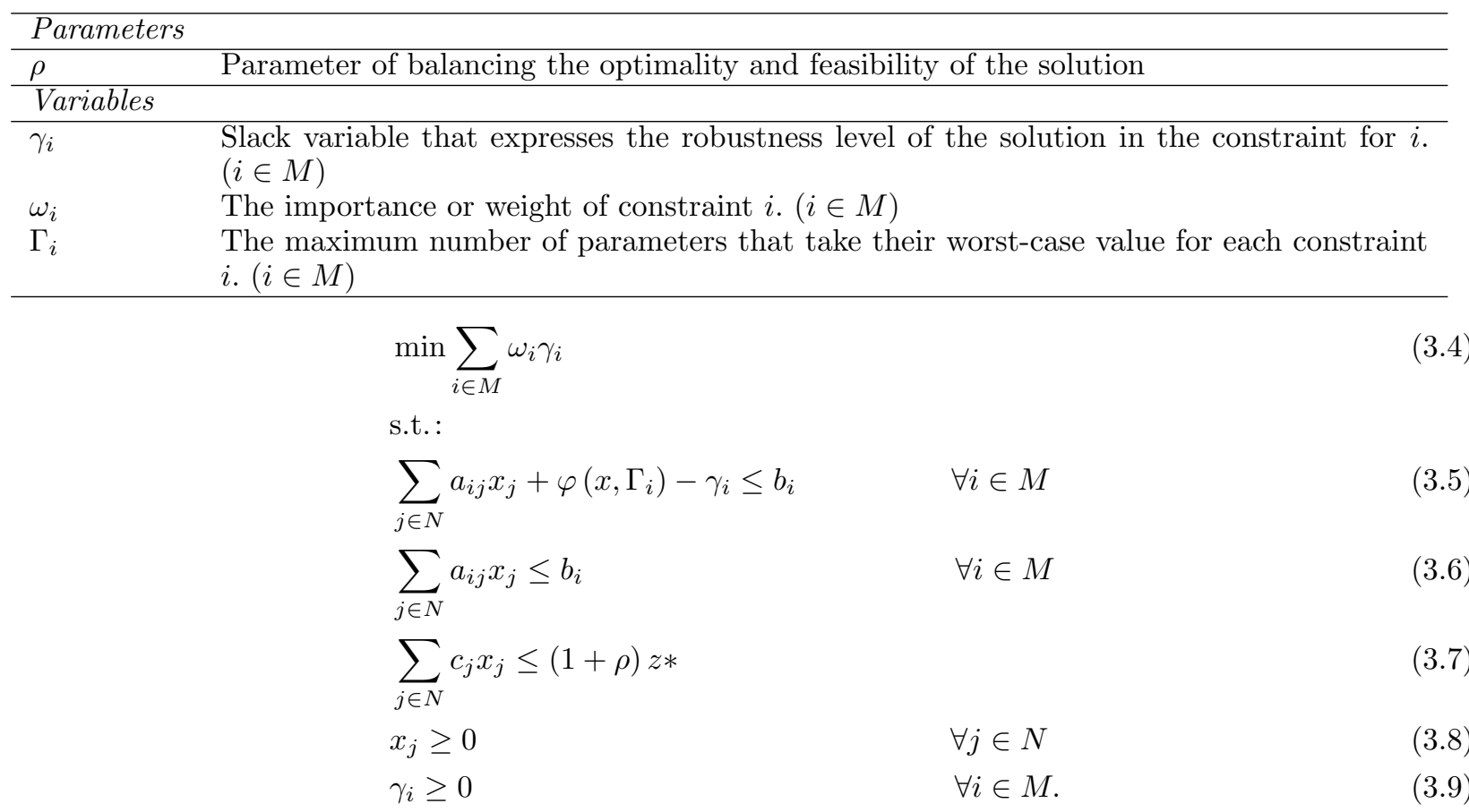

Objective function (3.4) minimizes the weighted sum of slack variables $\gamma_{i}$. Each variable $\gamma_{i}$ expresses the robustness level of the solution in constraint (3.5). Especially, $\gamma_{i}$ takes a positive value when the corresponded robust constraint $i$ is exceeded. Also, $\varphi\left(x, \Gamma_{i}\right)$ is the protection function which can be defined as follows:

$$
\varphi\left(x, \Gamma_{i}\right)=\operatorname{Max}_{S \subseteq N,|S| \leq \Gamma_{i}} \sum_{j \in S} \hat{a}_{i j} x_{i j} .
$$

As mentioned earlier, parameter $\rho$ is determined to balance the optimality and feasibility of the solution. $\rho=\infty$ demonstrates that the objective function of nominal problem is not considered and $\rho=0$ defines the nominal objective function. In constraint (3.7), a maximum deviation for the value of objective function is considered with respect to $z^{*}$ determined as the optimal objective function of nominal problem. In the objective function (3.4), $\omega_{i}$ are weights to consider different scales of constraints. LR counterpart of (3.1)-(3.3) can be defined as follows:

$$
\min \sum_{i \in M} \omega_{i} \gamma_{i}
$$

s.t. : 


$$
\begin{array}{ll}
\sum_{j \in N} a_{i j} x_{j}+\Gamma_{i} \alpha_{i}+\sum_{j \in N} \beta_{i j}-\gamma_{i} \leq b_{i} & \forall i \in M \\
-\hat{\mathrm{a}}_{i j} x_{j}+\alpha_{i}+\beta_{i j} \geq 0 & \forall i \in M, j \in N \\
\alpha_{i} \geq 0 & \forall i \in M \\
\beta_{i j} \geq 0 & \forall i \in M, j \in N \\
\sum_{j \in N} c_{j} x_{j} \leq(1+\rho) z * & \\
x_{j} \geq 0 & \forall j \in N \\
\gamma_{i} \geq 0 & \forall i \in M .
\end{array}
$$

This paper presents a light robust supply chain redesign model that incorporates varies sources of uncertainty. Unlike most of the published robust supply chain network design and redesign models that apply scenario-based robust optimization methods, we adapt the robust formulation with interval data uncertainty developed by Fischetti and Monaci [13] to overcome the aforementioned limitations of scenario-based models (e.g., dependency on the characteristics of scenarios specified as well as the computational overhead for managing a large number of scenarios). Light robustness is a new flexible method that can generate less conservative solutions in terms of objective function in comparison to other interval robust methods.

\subsection{Light robust supply chain network redesign with routing consideration (LRSCNRR)}

In real supply chain cases, demand and many operational costs are generally uncertain parameters. In this section, we extend the deterministic model presented in Section 2 to a robust optimization model in which demand, production cost, fixed establishing cost and saved cost of phasing-out are considered as uncertain parameters. The uncertain parameters $\tilde{d}_{i, p}, p \tilde{c}_{p}, \tilde{f} \tilde{b}_{i}, \tilde{f}_{i}$ are bounded and distributed in their correspondent intervals with the nominal values of $d_{i, p}, p c_{p}, f b_{i}, f s_{i}$ and the maximum variations of $\hat{d}_{i, p}, p \hat{c}_{p}, f \hat{b}_{i}$, and $\hat{f s_{i}}$ respectively. For example, $\tilde{d}_{i, p}$ belongs to the interval $\left[d_{i, p}-\hat{d}_{i, p}, d_{i, p}+\hat{d}_{i, p}\right]$. Also, we define parameter $\rho$ as a balancing factor to control the maximum deviation of optimal solution. The proposed light robust model is as follows:

$$
\operatorname{Min} \mathrm{Z}=\sum_{i \in k} \sum_{p \in p} \omega_{i p} \gamma_{i p}
$$

Subject to:

$$
\begin{gathered}
\sum_{i \in w_{e}} \sum_{j \in w} r_{i j} z_{i j}+\sum_{i \in w_{n}} f b_{i} z_{i i}+\Gamma^{f b} \alpha^{f b}+\sum_{i \in w_{n}} \beta_{i}^{f b}+\sum_{p \in p} \sum_{j \in w} f c_{j p} \sum_{i \in w_{e}} \operatorname{cap}_{i p} z_{i j}+\sum_{j \in w} f m_{j} z_{j j} \\
-\sum_{i \in w_{e}}\left[f s_{i}\left(1-\sum_{j \in w} z_{i j}\right)+f n_{i} \sum_{\substack{j \in w_{e} \\
i \neq j}} z_{i j}\right]+\Gamma^{f s} \alpha^{f s}+\sum_{i \in w_{e}} \beta_{i}^{f s}+\sum_{i \in f} \sum_{j \in w} \sum_{v \in v} u c_{v} x_{i j v}^{1} \\
+\sum_{i, j \in f \cup w} \sum_{v \in v} v c_{v} \operatorname{dis}_{i j} x_{i j v}^{1}+\sum_{i \in w} \sum_{j \in k} \sum_{v \in v} u c_{v} x_{i j v}^{2}+\sum_{i, j \in w \cup k} \sum_{v \in v} v c_{v} \operatorname{dis}_{i j} x_{i j v}^{2}+\sum_{i \in f} \sum_{j \in w} \sum_{p \in p} \sum_{v \in v} p c_{p} Q_{i j p v}^{1} \\
+\Gamma^{p c} \alpha^{p c}+\sum_{p \in p} \beta_{p}^{p c}+\sum_{i \in w} \sum_{p \in p} o c_{p} y_{i p}+\sum_{i \in w} \sum_{j \in k} \sum_{p \in p} \sum_{v \in v} h c_{i p} Q_{i j p v}^{2} \leq(1+\rho) c o * \\
\alpha^{f b}+\beta_{i}^{f b} \geq \hat{f} b_{i} z_{i i} \\
\alpha^{f s}+\beta_{i}^{f s} \geq \hat{f} s_{i}\left(\sum_{j \in w} z_{i j}-1\right) \\
\forall i \in w_{n}
\end{gathered}
$$




$$
\begin{array}{ll}
\alpha^{p c}+\beta_{p}^{p c} \geq \sum_{i \in f} \sum_{j \in w} \sum_{v \in v} \hat{p} c_{p} Q_{i j p v}^{1} & \forall p \in p \\
\sum_{v \in v} \sum_{j \in w \cup k} Q_{i j p v}^{2}-\sum_{v \in v} \sum_{j \in w \cup k} Q_{j i p v}^{2}+d_{i p}+\Gamma_{i p}^{d} \alpha_{i p}^{d}+\beta_{i p}^{d}-\gamma_{i p} \leq 0 & \forall i \in k, \forall p \in p \\
\alpha_{i p}^{d}+\beta_{i p}^{d} \geq \hat{d}_{i p} & \forall i \in k, \forall p \in p,
\end{array}
$$

and constraints (2.2)-(2.14) and (2.16)-(2.18)

$$
\alpha_{i p}^{d}, \alpha^{p c}, \alpha^{f b}, \alpha^{f s}, \beta_{i p}^{d}, \beta^{p c}, \beta^{f b}, \beta^{f s}, \gamma_{i p} \geq 0 .
$$

Equation (3.18) displays the objective function. Reduction in $\gamma_{i p}$ leads to controlling constraint (3.23). Simply put, when uncertain parameters $d_{i p}$ substantially deviate from the nominal values, equation (3.18) avoids infeasibility. Thus, the objective function attempts to reduce the infeasibility of the generated solutions under future variations. Constraint (3.19) controls the quality of generated solutions. It ensures that generated solutions have acceptable deviation from optimal solution of the nominal model which is displayed by $c o^{*}$. Also, it includes the other uncertain parameters. Constraints (3.20)-(3.22) and (3.24) are relevant to the robust optimization model. The rest of the constraints are similar to the constraints of the deterministic model.

\subsection{Revised light robust supply chain network redesign with routing consideration (RLRSCNRR)}

In this section, a revision on the application of the light robust approach in SCNRR is presented. Objective function (3.18) tries to minimize the total violation of a set of constraints (2.2)-(2.14), (2.16)-(2.18) and (3.19)-(3.25). It could be inferred that the LRSCNRR does not lead to a solution which distributes the capacity to demand points evenly. Such solution can increase the probability of shortage. By introducing the objective function (3.26) and replacing (3.18), in addition to considering the importance of each product in each demand point, the model tries to find a solution which distributes the available capacity to demand points evenly.

$$
\min \max \left\{\omega_{i p} \gamma_{i p}\right\} .
$$

In order to linearize (3.26), the objective function (3.27) subjected to (3.28) is introduced:

$$
\begin{aligned}
& \min R \\
& R \geq \omega_{i p} \lambda_{i p} .
\end{aligned}
$$

In the next section, the performance of the introduced objective function compared to the original one is illustrated.

\section{Computational Results}

This section aims to extend three significant purposes. First, a numerical example is generated to test the validation of the deterministic nominal model. Then, this numerical example will be solved using robust approaches to analyze the features of the presented robust model. At the end of this section, The models are solved using GAMS 23.5/CPLEX 12.2 on a computer with a core i5 $2.53 \mathrm{GHz}$ processor and 6GBs of RAM.

\subsection{Numerical example of DSCNRR model}

Assume a network with two factories $(A, B)$ that both can produce two types of products. There are two existing warehouses $\left(W_{1}, W_{2}\right)$ and one potential site to establish a new warehouse $\left(W_{3}\right)$. Moreover, there are five customer zones with determined demands and four vehicles in different types to be used for product transportations. Capacities, demands, distances and cost parameters are detailed in Appendix A. The cost 


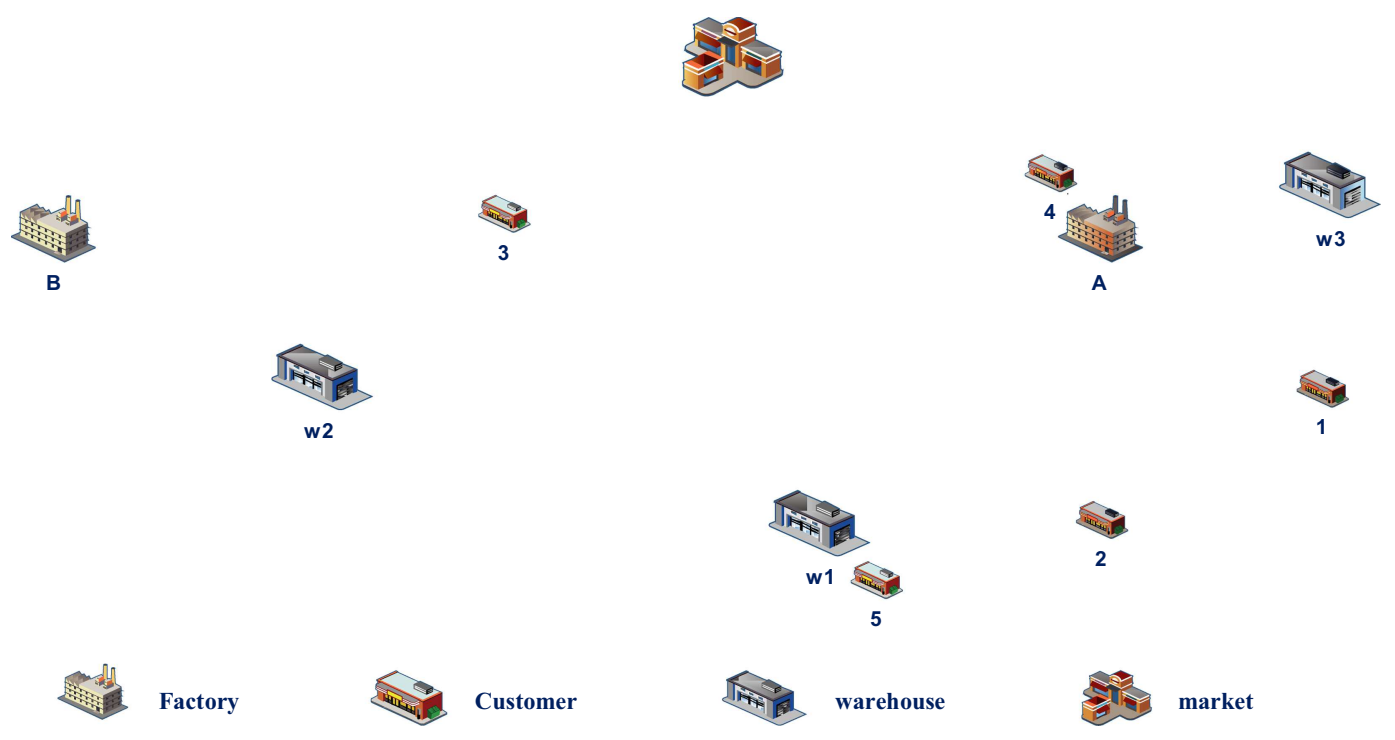

FiguRE 1. Network of numerical example.

parameters of this example are similar to cost parameters of the real-world problem in Melachrinoudis and Min [22]. Other parameters have been generated randomly.

The relocation costs depend heavily on the capacity of the existing warehouse at site $i$ that is being relocated $\left(\operatorname{cap}_{i, p}\right)$ as well as the distance from the current location to site $j\left(\operatorname{dis}_{i, j}\right)$ at which is relocated (consolidated). In addition, there is a fixed cost of relocation that is independent of capacity and distance while dependent on sites $i$ and $j,\left(r f_{i j}\right)$ [22]. Therefore, the cost of moving the capacity of warehouse $i$ to warehouse $j$ is:

$r_{i j}=r f_{i j}+\left(r c_{i j}+r c d \cdot \operatorname{dis}_{i j}\right) \operatorname{cap}_{i} \forall i \in W_{e}, j \in W$, where $r f_{i j}$ is a fixed cost of relocation that is independent of capacity and distance but dependent on sites $j$ and $i . r c_{i j}$ is the relocation cost per unit of capacity and $r c d$ is the cost of moving a unit of capacity one mile away from the current location [22].

Also, the savings correspond to the closing warehouse $i$ including the fixed cost, $f n_{i}$, the capacity cost and the profits from the sale of the assets, prorated per year of the planning horizon, $f s_{i}=f n_{i}+\zeta \operatorname{cap}_{i} \forall i \in W_{e}$, $\mathrm{w} \zeta$ indicates the savings per unit of capacity released.

After solving the problem by GAMS software in the aforementioned characteristics Figure 2 illustrates the final solution.

As shown in the Figure 2 the warehouse $W_{2}$ is phased-out, the new warehouse $W_{3}$ is established and the capacity of warehouse $W_{1}$ is transferred to the $W_{3}$. Also, the optimal routs in both of the echelons are specified by different colors. Total demand of product type $P_{2}$ is over than total production capacity of this product, so in the optimal solution 1000 units of product type $P_{2}$ is outsourced for warehouse $W_{1}$. The optimal cost of this problem is $7337042 \$$.

\subsection{Numerical example of RLRSCNRR model}

Due to the aforementioned characteristics of robust method, we can define parameter $\psi$ which displays the fraction of parameters that reach their worst values, $0 \leq \psi \leq 1$. According to this description we can define $\Gamma^{p c}=\psi|P|$ and so for other uncertain parameters. On the other hand, to determine range of uncertainty in non-deterministic parameters, it is defined that $p \hat{c}_{p}=\theta p c_{p}$ in which $0 \leq \theta \leq 1$ and so for other uncertain parameters. 


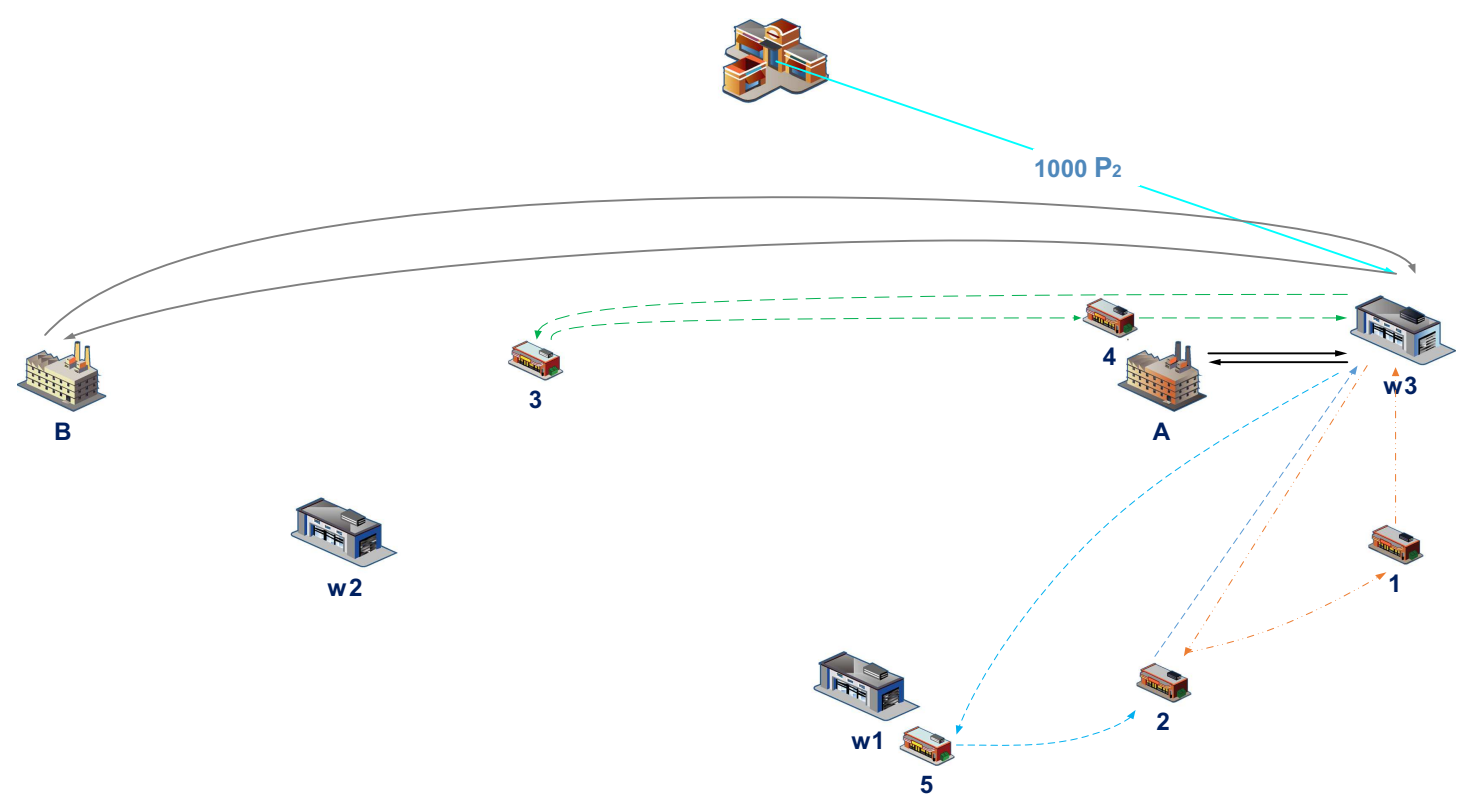

FIGURE 2. Optimal network of numerical example for DSCNRR.

It should be noted that the left hand side of constraint (3.19) is named as RLRCO. The RLRCO is real total cost achieved by the RLRSCNRR model.

$$
\begin{aligned}
\mathrm{RLRCO}= & \sum_{i \in w_{e}} \sum_{j \in w} r_{i j} z_{i j}+\sum_{i \in w_{n}} f b_{i} z_{i i}+\Gamma^{f b} \alpha^{f b}+\sum_{i \in w_{n}} \beta_{i}^{f b}+\sum_{p \in p} \sum_{j \in w} f c_{j p} \sum_{i \in w_{e}} \operatorname{cap}_{i p} z_{i j}+\sum_{j \in w} f m_{j} z_{j j} \\
& -\sum_{i \in w_{e}}\left[f s_{i}\left(1-\sum_{j \in w} z_{i j}\right)+f n_{i} \sum_{\substack{j \in w_{e} \\
i \neq j}} z_{i j}\right]+\Gamma^{f s} \alpha^{f s}+\sum_{i \in w_{e}} \beta_{i}^{f s}+\sum_{i \in f} \sum_{j \in w} \sum_{v \in v} u c_{v} x_{i j v}^{1}+\sum_{i, j \in f \cup w} \sum_{v \in v} v c_{v} \operatorname{dis}_{i j} x_{i j v}^{1} \\
& +\sum_{i \in w} \sum_{j \in k} \sum_{v \in v} u c_{v} x_{i j v}^{2}+\sum_{i, j \in w \cup k} \sum_{v \in v} v c_{v} \operatorname{dis}_{i j} x_{i j v}^{2}+\sum_{i \in f} \sum_{j \in w} \sum_{p \in p} \sum_{v \in v} p c_{p} Q_{i j p v}^{1}+\Gamma^{p c} \alpha^{p c}+\sum_{p \in p} \beta_{p}^{p c}+\sum_{i \in w} \sum_{p \in p} o c_{p} y_{i p} \\
& +\sum_{i \in w} \sum_{j \in k} \sum_{p \in p} \sum_{v \in v} h c_{i p} Q_{i j p v}^{2}
\end{aligned}
$$

In addition, RLROF is the objective function value of the RLRSCNRR model (3.18).

We consider $\theta$ as the range of uncertainty is equal to $30 \%, \psi$ is equal to $40 \%$ and the maximum deviation, $\rho$, is equal to $30 \%$. Also, it is assumed that all customers have same importance so that $\omega_{i p}=1$ for all $i$.

After solving the problem by gams software in the aforementioned characteristics Figure 3 illustrates the final solution. The optimal network of this supply chain in this condition is shown in Figure 3. As shown in the Figure 3 the capacity of warehouse $W_{1}$ is transferred to $W_{2}$, and the new warehouse $W_{3}$ is not established in this situation. The optimal routes in both of echelons are specified by different types of lines. With respect to uncertain demands, the amount of outsourced products is increased so that 20000 units of product $P_{1}$ and 30305 units of $P_{2}$ are outsourced. Also, due to aforementioned characteristics of the revised light robust approach, the model is allowed to ignore some portion of demand to control constraint (3.19). $\gamma_{i p}$ represents the ignored demand of each product for each customer. In this case, optimal solution leads to ignore 22400 units of product 


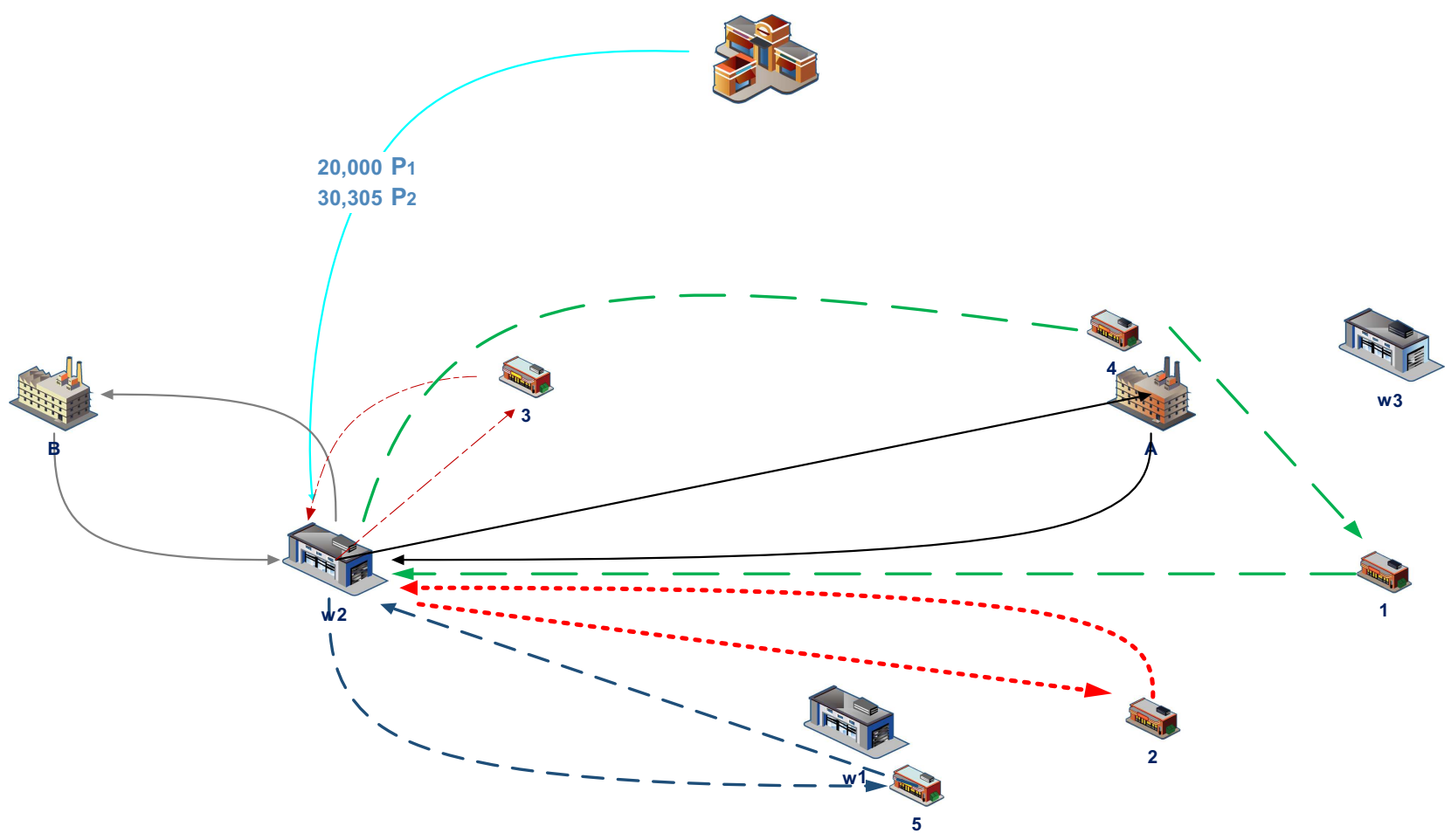

FIGURE 3. Optimal network of numerical example for RLRSCNRR.

$P_{1}$ and 45996 units of product $P_{2}$ for customer 1 . So, RLROF is equal to 68396 and RLRCO (cost of the $R L R S C N R R$ model) in aforementioned situation of parameters is equal to $9538155 \$$.

\subsection{Solution robustness analysis}

In this section, the robustness of different solutions obtained from three models (Deterministic, Light Robust, and Revised Light Robust) is compared. In order to do so, a simulation procedure is employed. In this procedure, each uncertain parameter is randomly generated in its own interval, as previously described. The solutions obtained from each of the three models are used to satisfy the demands in the generated circumstance. In order to compare the robustness of the three solutions, total unsatisfied demands of customers are calculated using each of the three solutions. This generation procedure is done by 1000 times and the total unsatisfied demands using each of the three solutions is calculated. Figure 4 represents the results of simulation and shows considerable decrease in number of unsatisfied demand.

Although, in some cases, using the DSCNRR solution may lead to even lower number of unsatisfied demands, it could be inferred that, in most cases using RLRSCNRR and LRSCNRR, solutions lead to much fewer unsatisfied demands. In order to illustrate the performance of the revised light robust model more explicitly, each 25 generating time is considered as a pack of generation, so that the total unsatisfied demand could be reported by the average of unsatisfied demands of these 25 generations. Figure 5 represents the results for 40 packs of generation (each of which includes 25 time generating). As is obvious, using the RLRSCNRR solution leads to much fewer unsatisfied demands which proves the good performance of the proposed revision in Section 3.3. 


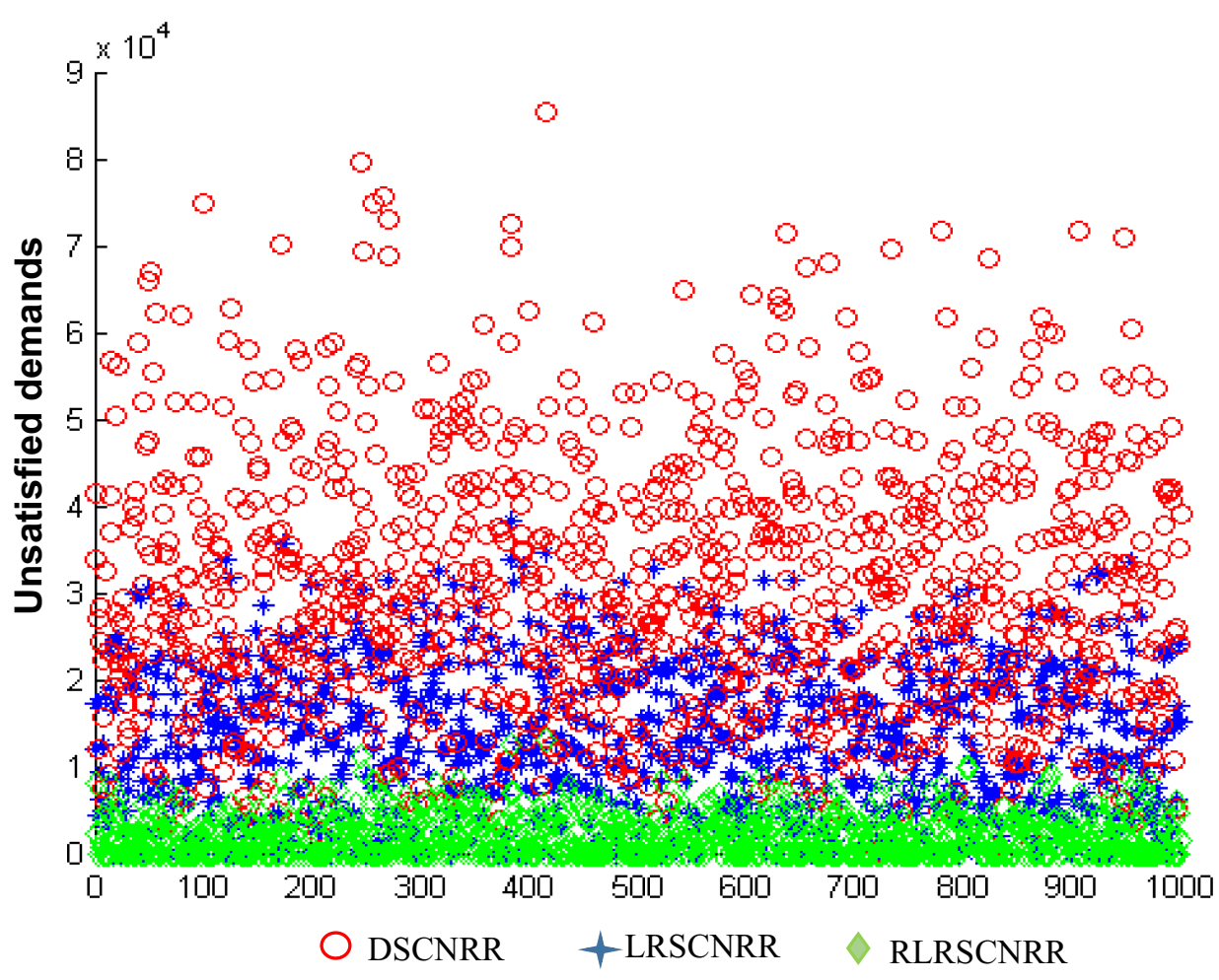

Figure 4. Amount of unsatisfied demands using three models.

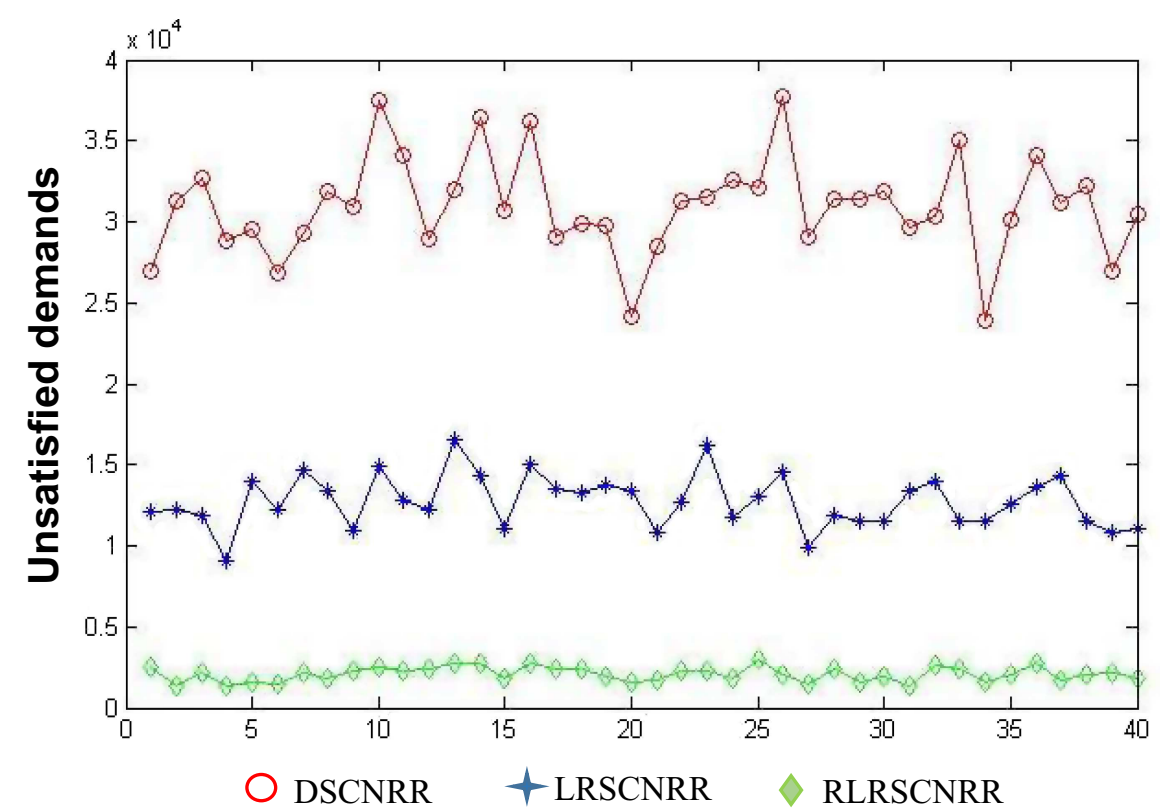

Figure 5. Amount of unsatisfied demands using three models (Packed). 


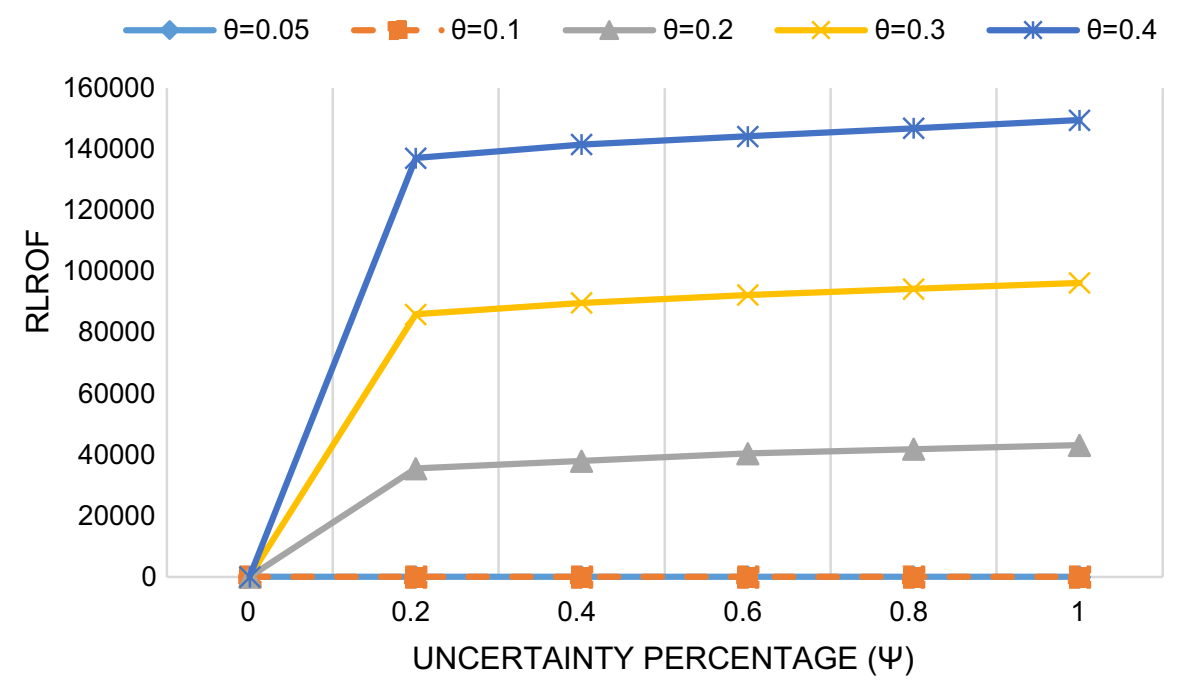

FiguRE 6. Effect of level of uncertainty on RLROF for the test problem in a fixed level of $\rho$.

TABLE 1. Effect of uncertainty for the test problem.

\begin{tabular}{|c|c|c|c|c|c|c|c|}
\hline \multicolumn{2}{|c|}{$\rho=0.2$} & \multicolumn{6}{|c|}{$\psi$} \\
\hline$\theta$ & Function & 0 & 0.2 & 0.4 & 0.6 & 0.8 & 1 \\
\hline \multirow[t]{2}{*}{0.05} & RLRCO & 7337042 & 7907675 & 7927675 & 7947383 & 7966799 & 7986215 \\
\hline & RLROF & 0 & 0 & 0 & 0 & 0 & 0 \\
\hline \multirow[t]{2}{*}{0.1} & RLRCO & 7337042 & 8568108 & 8590108 & 8612020 & 8633844 & 8655668 \\
\hline & RLROF & 0 & 0 & 0 & 0 & 0 & 0 \\
\hline \multirow[t]{2}{*}{0.2} & RLRCO & 7337042 & 8804451 & 8804451 & 8804451 & 8804451 & 8804451 \\
\hline & RLROF & 0 & 35498.4 & 37946.5 & 40382.8 & 41827.2 & 43140.7 \\
\hline \multirow[t]{2}{*}{0.3} & RLRCO & 7337042 & 8804451 & 8804451 & 8804451 & 8804451 & 8804451 \\
\hline & RLROF & 0 & 85945.2 & 89670.9 & 92284.8 & 94255 & 96225.1 \\
\hline \multirow[t]{2}{*}{0.4} & RLRCO & 7337042 & 8804451 & 8804451 & 8804451 & 8804451 & 8804451 \\
\hline & RLROF & 0 & 137069.4 & 141533.3 & 144184.1 & 146810.9 & 149485.4 \\
\hline
\end{tabular}

\subsection{Analysis for the robust optimization model}

In this part, two types of analyses will be examined to evaluate the changes of RLROF in the RLRSCNRR model. First, we will change uncertainty percentage $(\psi)$ and the range of uncertainty $(\theta)$ in a fixed allowed maximum deviation $(\rho)$, then we will change uncertainty percentage and the allowed maximum deviation in a fixed range of uncertainty.

Figure 6 represents the effect of level of uncertainty on the robust objective function in a fixed maximum deviation $\rho=0.2$ in different ranges of uncertainty, which are specified by colors and marks. The details of this chart are given in Table 1. (Note that in Fig. 6 the line of $\theta=0.1$ is covering the line of $\theta=0.05$ ).

The third column in Table $1(\psi=0)$ represents the deterministic problem which include no uncertain parameter, so RLRCO is equivalent to $c o^{*}$. In the other columns RLRCO is equal to the upper bound of constraint which is $(1+\rho) c o *$. Raising the value of $\theta$ (the range of uncertainty) in each column leads to rising the value of RLROF. Moreover in a fixed value for range of uncertainty, raising the value of $\psi$ (uncertainty percentage) leads 


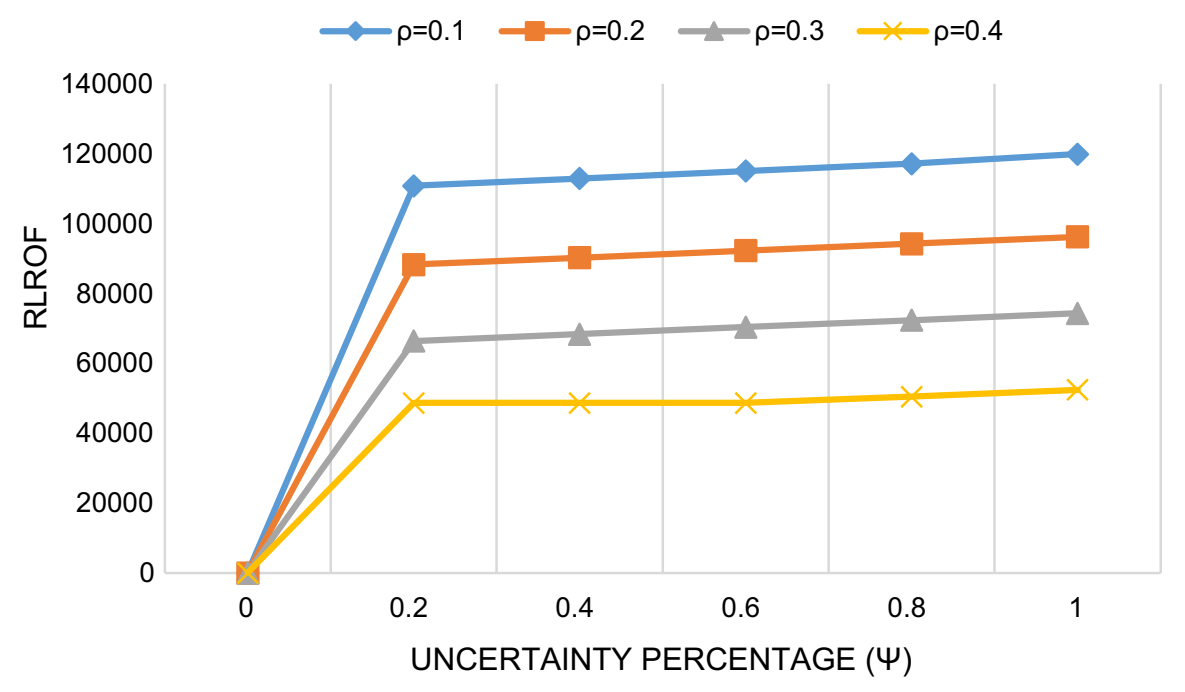

FIgURE 7. Effect of level of uncertainty on RLROF for the test problem in a fixed level of $\theta$.

TABLE 2. Effect of uncertainty for the test problem.

\begin{tabular}{|c|c|c|c|c|c|c|c|}
\hline \multicolumn{2}{|c|}{$\theta=0.3$} & \multicolumn{6}{|c|}{$\psi$} \\
\hline$\rho$ & Function & 0 & 0.2 & 0.4 & 0.6 & 0.8 & 1 \\
\hline \multirow[t]{2}{*}{0.1} & RLRCO & 7337042 & 8070746 & 8070746 & 8070746 & 8070746 & 8070746 \\
\hline & RLROF & 0 & 110845 & 112964.7 & 115065.6 & 117147.6 & 119894.8 \\
\hline \multirow{2}{*}{0.2} & RLRCO & 7337042 & 8804451 & 8804451 & 8804451 & 8804451 & 8804451 \\
\hline & RLROF & 0 & 88290.8 & 90296.8 & 92284.8 & 94255 & 96225.1 \\
\hline \multirow[t]{2}{*}{0.3} & RLRCO & 7337042 & 9538155 & 9538155 & 9538155 & 9538155 & 9538155 \\
\hline & RLROF & 0 & 66389.2 & 68395.2 & 70383.2 & 72353.4 & 74323.5 \\
\hline \multirow[t]{2}{*}{0.4} & RLRCO & 7337042 & 10271859 & 10271859 & 10271859 & 10271859 & 10271859 \\
\hline & RLROF & 0 & 48700 & 48700 & 48700 & 50451.8 & 52421.9 \\
\hline
\end{tabular}

to rising the value of RLROF. In this table because of the fixed amount of $\rho$, when RLROF takes positive values, RLRCO will be equivalent to $(1+\rho) c o *$. If $\mathrm{RLROF}=0, \mathrm{RLRCO}$ will take values between $c o^{*}$ and $(1+\rho) c o *$.

Figure 7 illustrates the effect of uncertainty level on the robust objective function in a fixed range of uncertainty $\theta=0.3$ in different values of maximum deviation of the optimal solution which are specified by colors and marks. The details of this chart (effect of changes in the value of $\psi$ and $\rho$ with fixed level of $\theta=30 \%$ on RLRCO and RLROF) are given in Table 2.

As is shown in Table 2, without considering uncertainty $(\psi=0)$ the RLRCO is equivalent to $c o^{*}$. While in the other columns $(\psi>0)$, RLRCO is equal to the upper bound which is $(1+\rho)$ co*. As, in each row, the value of $\rho$ is fixed, RLRCO will be equal to $(1+\rho)$ co* if RLROF takes positive values. Also, if RLROF=0, RLRCO will take values between $c o^{*}$ and $(1+\rho) c o *$.

In each column, taking a greater value of $\rho$ leads to reducing the value of RLROF. Furthermore, in a fixed value of maximum deviation raising the value of $\psi$ (uncertainty percentage) leads to rising the value of RLROF. 
TABLE 3. Unsatisfied demands in three approaches.

\begin{tabular}{|c|c|c|c|c|c|c|}
\hline \multirow[t]{2}{*}{ Test number } & \multicolumn{3}{|c|}{ Test size } & DSCNRR & LRSCNRR & RLRSCNRR \\
\hline & $\begin{array}{l}\text { Number of } \\
\text { plants }\end{array}$ & $\begin{array}{l}\text { Number of } \\
\text { warehouses }\end{array}$ & $\begin{array}{l}\text { Numbers of } \\
\text { customers }\end{array}$ & \multicolumn{3}{|c|}{ Unsatisfied demand $\left(\times 10^{4}\right)$} \\
\hline 1 & 2 & 3 & 5 & 3.125 & 1.303 & 0.160 \\
\hline 2 & 2 & 3 & 6 & 3.820 & 1.626 & 0.187 \\
\hline 3 & 2 & 3 & 7 & 4.415 & 1.708 & 0.199 \\
\hline 4 & 2 & 4 & 7 & 4.312 & 1.678 & 0.191 \\
\hline 5 & 2 & 4 & 8 & 4.632 & 1.712 & 0.200 \\
\hline
\end{tabular}

\subsection{Quality of solution}

In this part, 4 more numerical examples are generated and solved in order to demonstrate the improvement in solution regarding to unsatisfied demands. In these 5 test instances, different combinations of number of facilities in each echelon are considered while for all test problems, $\theta=30 \%, \psi=40 \%$, and $\rho=30 \%$.

From the Table 3 it could be inferred that unsatisfied demands in all test instances are decreased considerably using RLRSCNRR approach in comparison with two other approaches.

\section{MANAGERIAL impliCATIONS}

The proposed model provides a number of managerial implications to support supply chain managers to consider all aspects of supply chain redesign such as routing limitations, production capacity and uncertainty of parameters. These issues were studied to help and guide network designer for reestablishing a proper network as follow:

1. The routing with consideration of split delivery system, as one of the real-world problems involved in supply chain costs, is balanced in total cost calculations.

2. Due to the production capacity in the real cases, considering of possibility of outsourcing can help to prevent infeasibility.

3. Considering a minimax objective function instead of frequent objective function of LR model can help reach better solutions.

\section{Conclusion}

In this paper, we proposed a new mathematical model to redesign a three-echelon supply chain network problem. The proposed model extends previous studies by integrating key features needed to capture real-world conditions. We imported routing constraints to the redesigning problem. Moreover, because of the production capacity limitation, we considered outsourcing feature to satisfy a big portion of demands.

Uncertainty in some parameters is one of the inseparable features of real-world cases. Therefore, we considered some parameters as uncertain and implemented a robust approach to deal with this uncertainty. The light robust approach is a compromise between the robustness and the quality of the solution. Light robustness is a new flexible method which can generate fewer conservative solutions in terms of objective function in comparison with other interval robust methods. In this paper, we revised the objective function of LR approach to reach a better solution which makes fewer unsatisfied demands.

We presented a numerical example for the RLRSCNRR model to validate it. Then, we made some analyses to prove that, when there are uncertain parameters, our new objective function makes better solutions in comparison with the LR model and the deterministic model. Also, we did sensitive analysis on the robust model and observed effects of changing the level of uncertainty, range of uncertainty and the maximum deviation on 
the objective function value and total cost. The results show that, in uncertain situations, the revised light robust model makes better solutions with fewer number of unsatisfied demands, which makes for lower costs.

With respect to these results, the proposed model can be used for every supply chain with different purposes to find the optimal new design of the supply chain in a more realistic situation.

In our future research, we intend to take more factors into account in the supply chain, such as the periodic capacity transition or transportation modes. In future work, we can also model a green or sustainable network redesign or consider a comprehensive routing inventory redesign model. On the other hand, more research is needed to design new solution approaches to solve the proposed model.

\section{Appendix A.}

TABle A.1. Customer's demands.

\begin{tabular}{lll}
\hline \hline & $P_{1}$ & $P_{2}$ \\
\hline$K_{1}$ & 33000 & 55500 \\
$K_{2}$ & 64000 & 71500 \\
$K_{3}$ & 80000 & 0 \\
$K_{4}$ & 0 & 79500 \\
$K_{5}$ & 71000 & 44500 \\
\hline
\end{tabular}

TABlE A.2. Costs of warehouses $(\$)$.

\begin{tabular}{|c|c|c|c|c|}
\hline & & $W_{1}$ & $W_{2}$ & $W_{3}$ \\
\hline \multicolumn{2}{|c|}{$f m_{i}(i \in W)$} & 180000 & 190000 & 200000 \\
\hline \multicolumn{2}{|c|}{$f n_{i}\left(i \in W_{e}\right)$} & 220000 & 250000 & - \\
\hline \multirow{2}{*}{\multicolumn{2}{|c|}{$\begin{array}{l}f s_{i}\left(i \in W_{e}\right) \\
f b_{i}\left(i \in W_{n}\right)\end{array}$}} & 520000 & 670000 & - \\
\hline & & - & - & 900000 \\
\hline \multirow[t]{2}{*}{$f c_{i p}(i \in W)$} & $P_{1}$ & 1.1 & 1.3 & 1.2 \\
\hline & $P_{2}$ & 1.3 & 1.5 & 1.3 \\
\hline \multirow[t]{2}{*}{$h c_{i p}(i \in W)$} & $P_{1}$ & 9 & 9.5 & 9 \\
\hline & $P_{2}$ & 10 & 11 & 10.5 \\
\hline
\end{tabular}

TABle A.3. Production and warehousing capacities.

\begin{tabular}{llll}
\hline \hline & & $P_{1}$ & $P_{2}$ \\
\hline$M p_{i}(i \in F)$ & $A$ & 140000 & 150000 \\
& $B$ & 140000 & 100000 \\
$\operatorname{cap}_{i}(i \in W)$ & $W_{1}$ & 150000 & 100000 \\
& $W_{2}$ & 150000 & 200000 \\
& $W_{3}$ & 160000 & 170000 \\
\hline
\end{tabular}


TABle A.4. Fixed cost of consolidating warehouses (\$).

\begin{tabular}{llll}
\hline \hline & $W_{1}$ & $W_{2}$ & $W_{3}$ \\
\hline$W_{1}$ & 0 & 422037 & 465875 \\
$W_{2}$ & 550852 & 0 & 589860 \\
\hline
\end{tabular}

TABLE A.5. Vehicle's parameters.

\begin{tabular}{lllll}
\hline \hline & $V_{1}$ & $V_{2}$ & $V_{3}$ & $V_{4}$ \\
\hline $\operatorname{vcap}_{v}$ & 180000 & 170000 & 170000 & 180000 \\
$v c_{v}(\$)$ & 0.5 & 0.6 & 0.6 & 0.5 \\
$u c_{v}(\$)$ & 300 & 200 & 200 & 300 \\
\hline
\end{tabular}

TABle A.6. Procurement cost of products (\$).

\begin{tabular}{lll}
\hline \hline & $P_{2}$ & $P_{1}$ \\
\hline$p c_{p}$ & 2 & 2.2 \\
$o c_{p}$ & 22.2 & 22.5 \\
\hline
\end{tabular}

TABLE A.7. Distances.

\begin{tabular}{lllllllllll}
\hline \hline & $A$ & $B$ & $W_{1}$ & $W_{2}$ & $W_{3}$ & $K_{1}$ & $K_{2}$ & $K_{3}$ & $K_{4}$ & $K_{5}$ \\
\hline$A$ & 0.00 & 69.01 & 82.98 & 64.90 & 15.23 & 44.27 & 76.01 & 39.01 & 5.39 & 88.46 \\
$B$ & 69.01 & 0.00 & 96.57 & 39.92 & 83.15 & 93.48 & 102.73 & 30.00 & 67.12 & 102.73 \\
$W_{1}$ & 82.98 & 96.57 & 0.00 & 57.63 & 92.70 & 50.45 & 17.72 & 84.65 & 87.48 & 6.32 \\
$W_{2}$ & 64.90 & 39.92 & 57.63 & 0.00 & 79.92 & 68.26 & 66.40 & 39.92 & 66.22 & 63.60 \\
$W_{3}$ & 15.23 & 83.15 & 92.70 & 79.92 & 0.00 & 48.00 & 83.36 & 53.24 & 16.03 & 97.72 \\
$K_{1}$ & 44.27 & 93.48 & 50.45 & 68.26 & 48.00 & 0.00 & 37.16 & 68.25 & 49.65 & 54.08 \\
$K_{2}$ & 76.01 & 102.73 & 17.72 & 66.40 & 83.36 & 37.16 & 0.00 & 85.87 & 81.01 & 18.60 \\
$K_{3}$ & 39.01 & 30.00 & 84.65 & 39.92 & 53.24 & 68.25 & 85.87 & 0.00 & 37.22 & 90.96 \\
$K_{4}$ & 5.39 & 67.12 & 87.48 & 66.22 & 16.03 & 49.65 & 81.01 & 37.22 & 0.00 & 93.06 \\
$K_{5}$ & 88.46 & 102.73 & 6.32 & 63.60 & 97.72 & 54.08 & 18.60 & 90.96 & 93.06 & 0.00 \\
\hline
\end{tabular}

\section{REFERENCES}

[1] E. Asadi, F. Habibi, S. Nickel and H. Sahebi, A bi-objective stochastic location-inventory-routing model for microalgae-based biofuel supply chain. Appl. Energy 228 (2018) 2235-2261.

[2] R.H. Ballou and J.M. Masters, Commercial software for locating warehouses and other facilities. J. Bus. Logist. 14 (1993) 71-107.

[3] M. Bashiri and E. Ghasemi, A selective covering-inventory-routing problem to the location of bloodmobile to supply stochastic demand of blood. Int. J. Ind. Eng. Prod. Res. 29 (2018) 147-158.

[4] M. Bashiri and H.R. Rezaei, Reconfiguration of supply chain? A two stage stochastic programming. Int. J. Ind. Eng. Prod. Res. 24 (2013) 47-58.

[5] D. Bertsimas and M. Sim, Robust discrete optimization and network flows. Math. Program. 98 (2003) $49-71$.

[6] D. Bertsimas and M. Sim, The price of robustness. Oper. Res. 52 (2004) 35-53.

[7] X. Bing, J. Bloemhof-Ruwaard, A. Chaabane and J. Van Der Vorst, Global reverse supply chain redesign for household plastic waste under the emission trading scheme. J. Clean. Prod. 103 (2015) 28-39 
[8] J. Cortinhal, J. Lopes and T. Melo, Redesigning a three-echelon logistics network over multiple time periods with transportation mode selection and outsourcing opportunities. In: Technical Reports on Logistics of the Saarland Business School No 7, Saarland University of Applied Sciences (2014).

[9] K. Govindan, M. Fattahi and E. Keyvanshokooh, Supply chain network design under uncertainty: A comprehensive review and future research directions. Eur. J. Oper. Res. 263 (2017) 108-41.

[10] M. Fattahi, K. Govindan and E. Keyvanshokooh, A multi-stage stochastic program for supply chain network redesign problem with price-dependent uncertain demands. Comput. Oper. Res. 100 (2017) 314-332.

[11] S. Fazayeli, A. Eydi and I.N. Kamalabadi, Location-routing problem in multimodal transportation network with time windows and fuzzy demands: Presenting a two-part genetic algorithm. Comput. Ind. Eng. 119 (2018) 233-246.

[12] M. Feitó-Cespón, W. Sarache, F. Piedra-Jimenez and R. Cespón-Castro, Redesign of a sustainable reverse supply chain under uncertainty. A case study. J. Clean. Prod. 151 (2017) 206-217.

[13] M. Fischetti and M. Monaci, Light Robustness. Springer, Berlin Heidelberg (2009).

[14] J. Jouzdani, M. Fathian, A. Makui and M. Heydari, Robust design and planning for a multi-mode multi-product supply network: A dairy industry case study. Oper. Res. (2018) 1-30.

[15] M. Khatami, M. Mahootchi and R.Z. Farahani, Benders' decomposition for concurrent redesign of forward and closed-loop supply chain network with demand and return uncertainties. Transp. Res. Part E: Logist. Transp. Rev. 79 (2015) 1-21.

[16] F. Kiya and H. Davoudpour, Stochastic programming approach to re-designing a warehouse network under uncertainty. Transp. Res. Part E: Logist. Transp. Rev. 48 (2012) 919-936.

[17] W. Klibi, A. Martel and A. Guitouni, The design of robust value-creating supply chain networks: A critical review. Eur. J. Oper. Res. 203 (2010) 283-293.

[18] Y.J. Lee, T. Baker and V. Jayaraman, Redesigning an integrated forward-reverse logistics system for a third party service provider: an empirical study. Int. J. Prod. Res. 50 (2012) 5615-5634.

[19] S. Majidi, S. Yaghoubi and A. Jokar, Fuzzy green vehicle routing problem with simultaneous pickup - delivery and time windows. RAIRO: OR $\mathbf{5 1}$ (2017) 1151-1176.

[20] C.L. Martins, M.T. Melo and M.V. Pato, Redesigning a food bank supply chain network, Part I: Background and mathematical formulation. In: Technical Reports on Logistics of the Saarland Business School No 10 (2016).

[21] E. Melachrinoudis and H. Min, The dynamic relocation and phase-out of a hybrid, two-echelon plant/warehousing facility: A multiple objective approach. Eur. J. Oper. Res. 123 (2000) 1-15.

[22] E. Melachrinoudis and H. Min, Redesigning a warehouse network. Eur. J. Oper. Res. 176 (2007) $210-229$.

[23] M.T. Melo, S. Nickel and F. Saldanha-da-Gama, An efficient heuristic approach for a multi-period logistics network redesign problem. Top 22 (2014) 80-108.

[24] M.T. Melo, S. Nickel and F. Saldanha-Da-Gama, A tabu search heuristic for redesigning a multi-echelon supply chain network over a planning horizon. Int. J. Prod. Econ. 136 (2012) 218-230.

[25] M.T. Melo, S. Nickel and F.S. Saldanha Da Gama, Dynamic multi-commodity capacitated facility location: A mathematical modeling framework for strategic supply chain planning. Comput. Oper. Res. 33 (2006) 181-208.

[26] S.M.J. Mirzapour Al-e-hashem, H. Malekly and M.B. Aryanezhad, A multi-objective robust optimization model for multiproduct multi-site aggregate production planning in a supply chain under uncertainty. Int. J. Prod. Econ. 134 (2011) $28-42$.

[27] A. Nadizadeh and B. Kafash, Fuzzy capacitated location-routing problem with simultaneous pickup and delivery demands. Transp. Lett. 11 (2019) 1-19.

[28] J.C. Paz, J.A. Orozco, J.M. Salinas, N.C. Buriticá and J.W. Escobar, Redesign of a supply network by considering stochastic demand. Int. J. Ind. Eng. Comput. 6 (2015) 521-538.

[29] M. Rabbani, R. Heidari and R. Yazdanparast, A stochastic multi-period industrial hazardous waste location-routing problem: Integrating NSGA-II and Monte Carlo simulation. Eur. J. Oper. Res. 272 (2018) 945-961.

[30] J. Razmi, A. Zahedi-Anaraki and M. Zakerinia, A bi-objective stochastic optimization model for reliable warehouse network redesign. Math. Comput. Model. 58 (2013) 1804-1813.

[31] M. Schiffer and G. Walther, Strategic planning of electric logistics fleet networks: A robust location-routing approach. Omega 80 (2018) 31-42.

[32] A.L. Soyster, Technical note - convex programming with set-inclusive constraints and applications to inexact linear programming. Oper. Res. 21 (1973) 1154-1157.

[33] D.D. Tönissen and J.J. Arts, Economies of scale in recoverable robust maintenance location routing for rolling stock. Transp. Res. Part B: Methodol. 117 (2018) 360-377.

[34] S. Zokaee, A. Jabbarzadeh, B. Fahimnia and S.J. Sadjadi, Robust supply chain network design: An optimization model with real world application. Ann. Oper. Res. 257 (2014) 15-44. 